\title{
Pediatric Pituitary Adenoma
}

\author{
Tetsuro TAMURA, Ryuichi TANAKA, Ken KORII and Hideko OKAZAKi \\ Department of Neurosurgery, Brain Research Institute, Niigata University, Niigata, Japan
}

Key words: Pituitary adenoma, Children, Short stature, Primary amenorrhea

(Endocrine Journal 47: S95-S99, 2000)

PITUITARY adenoma in childhood and adolesence are rare. There are some reports about pediatric pituitary adenomas [1-9], but none are reported from Japan. In this paper we investigated the records of pediatric patients under 18 years old and discuss their clinical characteristics.

\section{Patients and methods}

The clinical charts of all patients at the University of Niigata from 1945 through December 1998 were retrospectively reviewed to identify pediatric patients with pituitary adenomas younger than 19 years of age at diagnosis. The adenomas were classified according to clinical phenotypes as defined by symptoms, serum hormone levels, and, if available, immunohistochemistry. A microadenoma was defined as a tumor $10 \mathrm{~mm}$ or less in diameter, and a giant adenoma was defined as a tumor more than $50 \mathrm{~mm}$ in diameter or reaching to foramen of Monro.

\section{Results}

The patient population is summarized in Table 1, grouped by tumor sub-type. The presenting symptoms and signs are summarized in Table 2. Age

Correspondense to: Tstsuro TAMURA, Department of Neurosurgery, Brain Research Institute, Niigata University, Asahimachi-dori 1-757, Niigata 951-8585, Japan

E-Mail: tamurate@npch.lamen.or.jp and sex distribution of the pediatric pituitary adenoma are summarized in Table 3, grouped by tumor sub-type. Tumor size is summarized in Table 4, grouped by tumor sub-type.

\section{Patient population}

Of the six hundred and seventy patients with pituitary adenomas between 1945 and 1998 in our institute, thirty three patients were younger than 19 years of age at diagnosis. There were eleven pediatric patients out of 146 patients (7.5\%) before 1977, when CT and RIA were introduced to our clinic. There were undetermined tumor phenotype whether non-functioning or prolactinoma, in six patients. From 1978 to 1998 , there were twenty two patients out of five hundred and twenty four patients $(4.2 \%)$.

\section{Presenting symptoms and signs}

Before 1978, all patients showed visual disturbance, and one girl showed sudden headache and vomiting with ophthalmoplegia that was compatible with pituitary apoplexy. Three boys were of tall stature, two patients were of short stature, and all five girls showed menstrual abnormality. Primary amenorrhea was presented in one patient with nonfunctioning adenoma and two with undetermined tumor phenotype.

From 1978, neurological deficit was present in 8 
Table 1. patient population out of all age

\begin{tabular}{lcc}
\hline tumor type & $1945-1977$ & $1978-1998$ \\
\hline Non-functioning adenoma & & $3 / 186(1.6 \%)$ \\
& $>8 / 117(6.8 \%)$ & \\
Prolactinoma & & $12 / 163(7.4 \%)$ \\
Giantism/Acromegaly & $3 / 28(10.7 \%)$ & $1 / 137(0.7 \%)$ \\
Cushing's disease & $0 / 1(0.0 \%)$ & $6 / 36(16.7 \%)$ \\
TSH secreting adenoma & $0 / 0$ & $0 / 2(0.0 \%)$ \\
\hline Total & $11 / 146(7.5 \%)$ & $22 / 524(4.2 \%)$ \\
\hline
\end{tabular}

Table 2. Symptoms and signs of pediatric pituitary adenomas

\begin{tabular}{lcc}
\hline & $1945-1977(\mathrm{n}=11)$ & $1978-1998(\mathrm{n}=22)$ \\
\hline Neurological & 11 & 7 \\
$\quad$ visual disturbance & 1 & 2 \\
$\quad$ ophthalmopregia & & 1 \\
$\quad$ consciousness disturbance & & 6 \\
Endocrinological & 3 & \\
$\quad$ Cushingoid & 2 & 1 \\
tall stature & 2 & 2 \\
acromegaly & $5 / 5$ & $9 / 10$ \\
growth retardation & $(3)$ & $(3)$ \\
menstrual abnormality & & 1 \\
(primary amenorrhea) & & \\
loss of libido & & \\
\hline
\end{tabular}

patients out of 22 patients. Short stature was shown in one boy with non-functioning adenoma and one with Cushing's disease. Primary amenorrhea was present in three patients with prolactinomas.

\section{Prolactinoma}

Twelve patients had prolactinomas; five were boys and seven were girls. One boy presented only loss of libido, but another four presented with visual disturbance. Six girls did not show any neurological deficit, and three of them showed primary amenorrhea. Four tumors were microadenomas, six were macroadenomas and two tumors of boys were giant adenomas. One girl with microadenoma was treated only with bromocriptine. Giant adenomas were partially removed through transcranial approach, and another nine tumors were removed transsphenoidally. Post-operative normal prolactin levels were achieved in six patients including one boy.

\section{Cushing's disease}

Six patients had ACTH secreting pituitary adenomas causing Cushing's disease. Two patients were in the prepubertal age group. The boy and girl ratio was $3: 3$. Three tumors were microadenomas including one not identified pathologically or visualized by the surgeon, and three were macroadenomas. One boy died soon after transsphenoidal surgery because of pre-existing respiratory failure. Four patients went into remission after transsphenoidal surgery, but one boy showed relapse biochemically after three years.

\section{Acromegaly/Giantism}

Before 1978, there were three tall boys and two of them had acromegalic features with eosinophilic adenomas, but after 1978, there was only one boy 
Table 3. Age and sex distribution of pediatric pituitary adenomas

1) before $1978(n=11)$

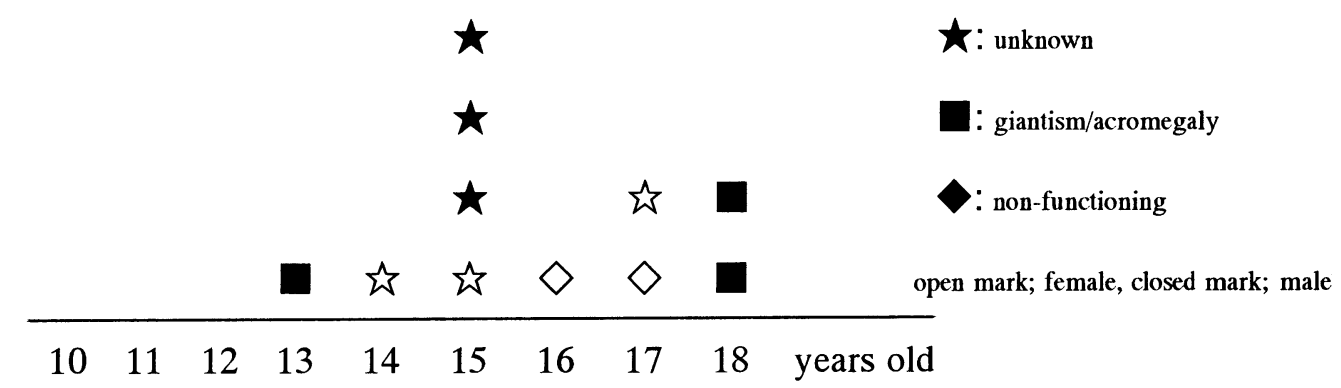

2) after $1978(n=22)$

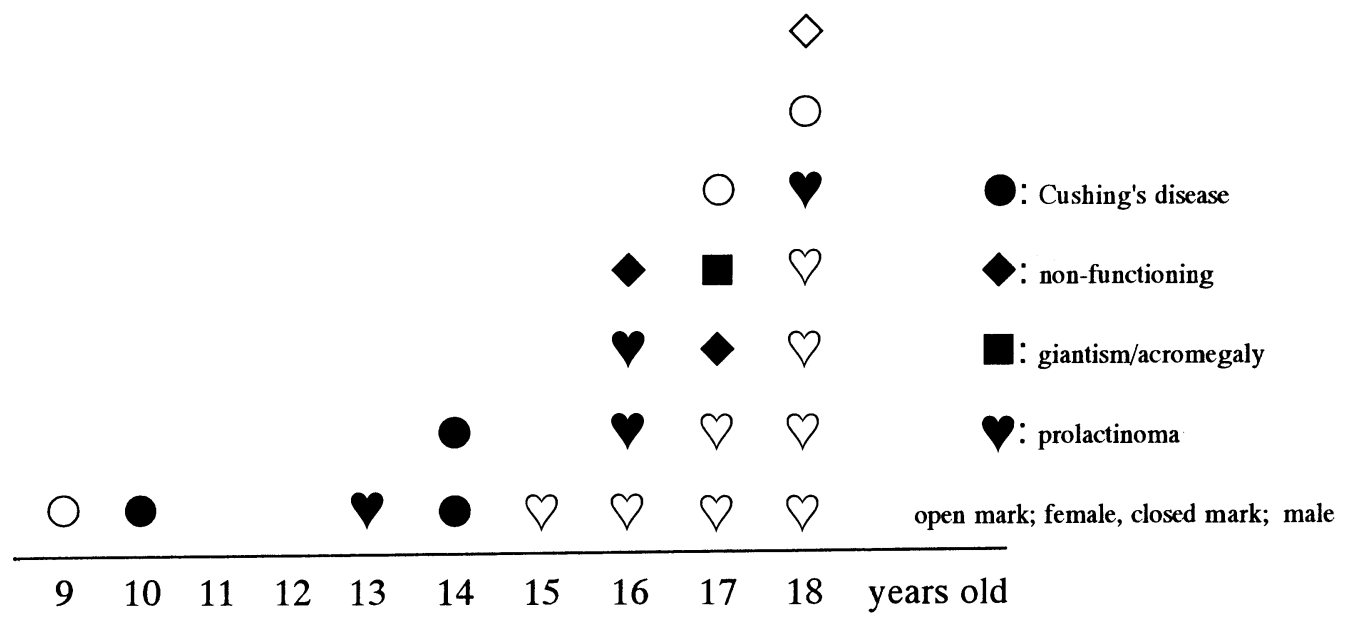

Table 4. Tumor size in pediatric pituitary adenomas after $1978(n=22)$

\begin{tabular}{lccc}
\hline tumor type & microadenoma & macroadenoma & giant adenoma \\
\hline Prolactinoma & 4 & 6 & 2 \\
Acromagely & 3 & 1 & \\
Cushing's disease & & 3 & 2 \\
Non-functioning adenoma & 7 & 11 & 4 \\
\hline Total & & & \\
\hline
\end{tabular}

presenting with acromegaly and he presented severe pituitary apoplexy and died because of internal carotid artery occlusion. In all four patients, serum GH level could not be determined before operation. One patient with acrogiantism had normal serum GH levels without paradoxical response to TRH 11 years after transcranial tumor resection and postoperative ${ }^{60} \mathrm{Co}$ irradiation.

\section{Non-functioning adenoma}

Before 1978, there were two definite female patients, six patients were not determined because serum prolactin measurement and immunohistochemistry could not be performed. All patients showed visual disturbance. One girl showed massive regrowth after her first operation and irradiation, and died soon after a second operation. In another 
female patient, non-functioning pituitary adenoma was controlled by operation and irradiation, but parasellar meningioma occurred and was removed after 18 years. After 1978, there were only three patients. One boy showed short stature, and he received GH replacement therapy after transsphenoidal partial removal and irradiation. One girl with giant adenoma showed panhypopituitarism preoperatively and another boy with giant adenoma became panhypopituitarism after operation.

\section{Discussion}

There are some reports about pituitary adenomas in childhood and adolesence. Pediatric pituitary adenomas constitute $2.1-6 \%$ of all pituitary adenomas treated surgically. In the pre CT-era, pediatric adenoma may have been slightly predominant (7.5\%) in incidence. The reason for this is that microadenoma, especially prolactin secreting and ACTH secreting adenomas, could not be diagnosed. In our series, like in other reports $[1,2,4-9]$, the frequency of clinically non-functioning adenomas differed distinctly from that of adults: this is very rare in childhood. Even though we cannot define non-functioning adenoma or prolactinoma before the CTera, these tumors may have been predominantly prolactinomas. Prepubertal children were most likely to have ACTH secreting adenomas, and postpubertal patients were most likely have prolactinomas. Gender differences were not observed in Cushing's disease and prolactinomas.

Menstrual abnormality was a frequent symptom in all tumor types. Large adenoma is predominant in pediatric age. Non-functioning adenoma can lead to menstrual irregularities. All prolactinomas inducing primary amenorrhea were macroadenomas. Primary amenorrhea was not observed after 19 years of age. Short stature occurred with non-functioning adenoma and Cushing's disease in our CT-era series, but it did not appear in the patients after 19 years of age, so it is meaningful to divide between the 18 and 19 year age groups. Non-functioning adenomas in pediatric age are extremely rare. Short stature in Cushing's disease is well recognized, but a few cases with non-functioning adenoma are also reported [10]. We have reported such a case contained in the current [11].

Eleven out of twelve patients with prolactinomas underwent surgery. Three boys and five girls underwent transsphenoidal surgery, and two boys underwent transcranial surgery. After transphenoidal surgery, one boy and five girls achieved normal prolactin levels, and three patients with primary amenorrhea achieved normal menstrual cycles. Postoperative bromocriptine therapy in persistent patients was useful to control tumor growth or normoprolactinemia, but in three boys, their tumors were bromocriptine resistant and they received irradiation with or without a second operation.

Four out of six patients (66.7\%) with Cushing's disease went into initial remission. Even though this is a small series, it is almost in accordance with adult series and another pediatric series [12, 13].

Incomplete sphenoid pneumatization was frequently recognized in pediatric patients, but the bone could be easily removed. Transsphenoidal surgery was thought to be the best treatment if the tumor was located mainly in the sella.

\section{Conclusion}

Our data provide basic information showing that the distribution of the various pituitary adenoma types differs from adults; prepubertal children are most likely to have an ACTH secreting adenoma causing Cushing's disease. Pediatric pituitary adenoma is predominant in functioning adenoma. Sex difference is insignificant in pediatric prolactinoma and Cushing's disease. Primary amenorrhea may indicate a pituitary adenoma and should prompt a neuro-imaging study of the sella. Short stature should raise clinical suspicion of a pituitary adenoma such as non-functioning adenoma. Early diagnosis and treatment should be performed.

\section{References}


2. Richmond IL, Wilson CB (1978) Pituitary adenomas in childhood and adolescence. J Neurosurg 49: 163168.

3. Rocco CD, Maira G, Borrelli P (1982) Pituitary microadenomas in children. Child's Brain 9: 165-178.

4. Fraioli B, Ferrante L, Celli P (1983) Pituitary adenomas with onset during puberty. $J$ Neurosurg 59: 590595.

5. Kanter SL, Mickele JP, Hunter SB, Rhoton AL (1985-86) Pituitary adenomas in pediatric patients: are they more invasive? Pediat Neurosci 12: 202-204.

6. Dyer EH, Civit T, Visot A, Delalande O, Derome P (1994) Transsphenoidal surgery for pituitary adenomas in children. Neurosurgery 34: 207-212.

7. Kane LA, Leinung MC, Scheithauer BW, Bergstralh EJ, Laws ER, Jr, Groover RV, Kovacs K, Horvath E, Zimmerman D (1994) Pituitary adenomas in childhood and adolescence. J Clin Endocrinol Metab 79: 1135-1140.
8. Partington MD, Davis DH, Laws ER,Jr, Scheithauer BW (1994) Pituitary adenomas in childhood and adolescence. Results of transsphenoidal surgery. $J$ Neurosurg 80: 209-216.

9. Mindermann T, Wilson CB (1995) Pediatric pituitary adenomas. Neurosurgery 36: 259-269.

10. Abe T, Ludecke DK, Saeger W (1998) Clinically nonsecreting pituitary adenomas in childhood and adolescence. Neurosurgery 42: 744-751.

11. Tamura T, Hasegawa K, Okazaki H, Morii K, Tanaka R (1998) Adlescent Nonfunctioning Pituitary Adenoma with Short Stature. Endocrine Journal 45 (suppl): S179-S181.

12. Leinung MC, Laws ER,Jr, Zimmerman D (1995) Long term follow-up of transsphenoidal surgery for the treatment of Cushing's disease in childhood. $J$ Clin Endocrinol Metab 80: 2475-2479.

13. Savage MO, Besser GM (1996) Cushing's disease in childhood. Trends Endocrinol Metab 7: 213-216. 